\title{
A New Time-Varying Feedback RISE Control of PKMs: Theory and Application
}

\author{
Hussein Saied ${ }^{1,2}$, Ahmed Chemori ${ }^{1}$, Mohamed Bouri ${ }^{3}$, Maher El Rafei ${ }^{2}$, Clovis Francis ${ }^{2}$ and Francois Pierrot $^{1}$
}

\begin{abstract}
In this paper, we propose a novel time-varying feedback control strategy based on the Robust Integral of the Sign of the Error (RISE). The main motivation is to enhance the tracking performance of RISE controller at high dynamic operating conditions. RISE control law ensures a semi-global asymptotic tracking without introducing severe restrictions on the uncertain and nonlinearly parametrized systems. More nonlinearities are added to the original RISE control law by replacing the static feedback gains with nonlinear ones which depend on the system state variables. The proposed contribution is implemented in real-time experiments on a non-redundant three-degrees-of-freedom parallel manipulator named Delta. Comparing to the standard RISE controller, experimental results show better tracking performances of the proposed time-varying feedback RISE controller.
\end{abstract}

\section{INTRODUCTION}

In the last decades, nonlinear control strategies have been studied extensively by researchers who show a wide interest in the design and stability analysis of nonlinear controllers [1]. The conventional linear control performance degrades while dealing with nonlinear systems and may lead to instability, while nonlinear control conserves the stability granting better global performance and robustness. On the one hand, one of the most studied nonlinear control algorithms in the literature is the usage of nonlinear functions as feedback gains instead of the static ones. The simple Proportional-Integral-Derivative (PID) controller was first improved towards a NonLinear PID (NLPID) controller in [2]. The feedback gains in such control type are adjusted online depending on the system states. On the other hand, gain-scheduling control approaches have been examined in [3], showing good robustness and ability to compensate uncertainties, but still considered complex for implementation because of their hard tuning process. Moreover, adaptive feedback control strategies have been also investigated in the literature [4]. Robust Integral of the Sign of the Error (RISE) is a nonlinear continuous control strategy developed recently in [5] for uncertain nonlinear systems, it is based on limited assumptions on the system dynamics. Lately, several RISE-based controllers have been developed and applied in real-time applications demonstrating the robustness and

\footnotetext{
${ }^{1}$ Hussein Saied, Ahmed Chemori and Francois Pierrot are with LIRMM, University of Montpellier, CNRS, Montpellier, France $\{$ saied, chemori, pierrot\}alirmm.fr

${ }^{2}$ Hussein Saied, Maher El Rafei and Clovis Francis are with CRSI, Lebanese University, Beirut, Lebanon \{maher.elrafei, cfrancis\}@ul.edu.lb

${ }^{3}$ Mohamed Bouri is with EPFL, Station 9, Lausanne CH-1015, Switzerland mohamed.bourieepfl.ch
}

disturbances-rejection provided by RISE feedback closedloop architecture [6]-[9]. In [10] and [11], RISE-based adaptive dynamics control schemes have been applied to Parallel Kinematic Manipulators (PKMs), were it has been the first and only time RISE control strategy is implemented to parallel robots, proving the good performance of the proposed controllers experimentally.

Control of parallel robots is considered a challenging task due to these factors: nonlinearities which may increase when operating at high speed, time-varying parameters, non-modelled phenomena and uncertainties. Several control solutions have been proposed for PKMs in the world of control and robotics. The simple linear PID controller [12], NLPID controller based on nonlinear feedback gains [13], Computed Torque Control [14], Ho control [15], PD control with computed feedforward [16], dual-space control [17], adaptive dynamics controllers [11], [18] and time-varying feedback controllers [19], [20] where authors got use of the nonlinear feedback gains to improve the global performance of original controllers.

In this paper, a new time-varying feedback RISE controller is proposed and applied experimentally to a parallel robot. RISE controller is a non-model-based feedback control robust against uncertainties, disturbances and unstructured nonlinearities which can be a good control solution of PKMs. This contribution takes the advantage of using nonlinear feedback gains that depend on the system states instead of static feedback gains as in the standard RISE control law, and without any prior knowledge about the model of the robot. Additional nonlinearity to RISE control may allow it to compensate for great percentages of the high nonlinearities abundant extensively in PKMs. The main motivation behind this work is to improve the global performance of the original RISE controller as well as control performance of PKMs in terms of precision, robustness towards payload variation and high-speed motions. In order to validate the relevance of the proposed controller, real time experiments are conducted on a three-degree-of-freedom (3-DOF) non-redundant PKM named Delta robot.

The remaining part of the paper is organized as follows. Section II introduces a brief background on RISE control law. The proposed control solution is described in section III. In section IV, the platform description and dynamic modeling are addressed. The experimental results are presented in section V. Section VI sums up the main drawn conclusions and proposes a future work. 


\section{BACKGROUND ON RISE CONTROL LAW}

As reported in [5], RISE control law is a full-state feedback tracking controller for a class of uncertain MIMO nonlinear systems. It was shown in [5] that RISE controller achieves semi-global asymptotic tracking under limited assumptions regarding the system nonlinearities.

Consider the high-order MIMO nonlinear system of the general form

$$
M\left(x, \dot{x}, \ldots, x^{(n-1)}\right) x^{(n)}+f\left(x, \dot{x}, \ldots, x^{(n-1)}\right)=u
$$

where $(.)^{(i)}(t)$ refers to the ith derivative with respect to time, $x^{(i)}(t) \in \mathbb{R}^{m}$ for $i=0, . ., n$ are the system states, $u(t) \in \mathbb{R}^{m}$ is the control input signal, $M(.) \in \mathbb{R}^{m \times m}$ and $f(.) \in \mathbb{R}^{m}$ are uncertain nonlinear functions. If $x, \dot{x} \in \mathscr{L}_{\infty}$ then $f($.$) is$ bounded, and the first and second partial derivatives of $M($. and $f($.$) with respect to x, \dot{x}$ exist and bounded. Let $M($.$) be$ a symmetric and positive-definite matrix bounded by

$$
\underline{m}\|\xi\|^{2} \leq \xi^{T} M(.) \xi \leq \bar{m}(.)\|\xi\|^{2}
$$

where $\underline{m}$ and $\bar{m}($.$) are positive constant, and positive non-$ decreasing function respectively. The reference trajectory $x_{d}(t) \in \mathbb{R}^{m}$ is chosen to be a $C^{n+2}$ function. The tracking error $e_{1}(t) \in \mathbb{R}^{m}$ is then defined as follows:

$$
e_{1}=x_{d}-x
$$

A set of auxiliary control signals $e_{i}(t) \in \mathbb{R}^{m}$ for $i=2,3, . ., n$ are given as follows:

$$
e_{i}=\dot{e}_{i-1}+e_{i-1}+e_{i-2}
$$

such that $e_{n}(t)$ is measurable since it is a function of the system states and reference trajectories. Then, RISE control law proposed in [5] is written as follows:

$$
\begin{aligned}
u(t) & =\left(K_{s}+I_{m}\right) e_{n}(t)-\left(K_{s}+I_{m}\right) e_{n}\left(t_{0}\right) \\
& +\int_{t_{0}}^{t}\left[\left(K_{s}+I_{m}\right) \Lambda e_{n}(\tau)+\beta \operatorname{sgn}\left(e_{n}(\tau)\right)\right] d \tau
\end{aligned}
$$

where $K_{s}, \Lambda, \beta \in \mathbb{R}^{m \times m}$ are positive-definite diagonal control gain matrices, $I_{m} \in \mathbb{R}^{m \times m}$ is the identity matrix, and $\operatorname{sgn}(.) \in$ $\mathbb{R}^{m}$ is defined as $\operatorname{sgn}(\varepsilon)=\left[\operatorname{sgn}\left(\varepsilon_{1}\right) \operatorname{sgn}\left(\varepsilon_{2}\right) \cdots \operatorname{sgn}\left(\varepsilon_{m}\right)\right] \forall \varepsilon=$ $\left[\begin{array}{llll}\varepsilon_{1} & \varepsilon_{2} & \cdots & \varepsilon_{m}\end{array}\right]$. The second term of equation (5) ensures a zero input signal at time $t_{0}$. The standard signum function used in RISE controller can hold smooth bounded disturbances for a sufficient condition on the feedback gain $\beta$. The stability analysis detailed in [5] shows that all the system signals are bounded under closed-loop operation and $e_{1}^{(i)}(t) \rightarrow 0$ as $t \rightarrow \infty$ with $i=0,1, \ldots, n$ where the control gain $K_{s}$ is selected sufficiently large.

\section{Proposed CONTRIBUtion: TIME-VARYING FEEDBACK RISE CONTROLLER}

It was reviewed in the introduction of this paper a series of control algorithms that were enhanced using the online adjustment of feedback gains through different techniques: nonlinear functions, gain-scheduling and adaptation. In order to boost up the tracking performance of the standard RISE controller, this work suggests to replace some of the static feedback gains with nonlinear varying ones.

The standard RISE equation of (5) can be divided into two parts: linear feedback $\left(\left(K_{s}+I_{m}\right) e_{n}(t)-\left(K_{s}+\right.\right.$ $\left.I_{m}\right) e_{n}\left(t_{0}\right)+\int_{t_{0}}^{t}\left[\left(K_{s}+I_{m}\right) \Lambda e_{n}(\tau) d \tau\right)$ and a nonlinear part $\left(\int_{t_{0}}^{t} \beta \operatorname{sgn}\left(e_{n}(\tau)\right) d \tau\right)$. By modifying the linear feedback gains $K_{s}, \Lambda$ into nonlinear functions $K_{s}(),. \Lambda($.$) , the control law$ could be more accommodated to nonlinear systems and compensates for more abundant nonlinearities.

The new time-varying feedback RISE control law is given as follows:

$$
\begin{aligned}
u(t) & =\left(K_{s}(.)+I_{m}\right) e_{n}(t)-\left(K_{s}(.)+I_{m}\right) e_{n}\left(t_{0}\right) \\
& +\int_{t_{0}}^{t}\left[\left(k_{s}+1\right) I_{m} \Lambda(.) e_{n}(\tau)+\beta \operatorname{sgn}\left(e_{n}(\tau)\right)\right] d \tau
\end{aligned}
$$

where $K_{s}(),. \Lambda(.) \in \mathbb{R}^{m \times m}$ are the nonlinear feedback gains defined as

$$
\begin{gathered}
K_{s}\left(e_{n}, \alpha_{K_{s}}, \delta_{K_{s}}\right)= \begin{cases}k_{s}\left|e_{n}\right|^{\alpha_{K_{s}}-1}, & \left|e_{n}\right|>\delta_{K_{s}} \\
k_{s} \delta_{K_{s}}^{\alpha_{K_{s}}-1}, & \left|e_{n}\right| \leq \delta_{K_{s}}\end{cases} \\
\Lambda\left(\int e_{n}, \alpha_{\Lambda}, \delta_{\Lambda}\right)= \begin{cases}\lambda\left|\int e_{n}\right|^{\alpha_{\Lambda}-1}, & \left|\int e_{n}\right|>\delta_{\Lambda} \\
\lambda \delta_{\Lambda}^{\alpha_{\Lambda}-1}, & \left|\int e_{n}\right| \leq \delta_{\Lambda}\end{cases}
\end{gathered}
$$

where $k_{s}, \alpha_{K_{s}}, \delta_{K_{s}}, \lambda, \alpha_{\Lambda}, \delta_{\Lambda}$ are the control parameters of the nonlinear feedback gains to be tuned.

The selection of $\alpha_{K_{s}}$ and $\alpha_{\Lambda}$ within the intervals $[0.5,1]$ and $[1,1.5]$ respectively produces the nonlinear gain behaviors depicted in Fig. 1. The nonlinear function $K_{s}($.$) gives$ high gain values for small combined errors $e_{n}$ and small gain values for large combined errors that could be resulting in a rapid transition of the closed-loop system states and favorable damping. Besides, $\Lambda($.$) attains high gain values$ for large steady state values of the combined error $e_{n}(\Lambda()$. varies as function of the integral of the combined error) and small gain values for the small steady state combined errors solving the integral windup problem by reducing the integral action when the combined error is large.

\section{Delta Robot: Description, Modeling AND CONTROL APPLICATION}

\section{A. Delta robot: description and kinematics}

Delta robot is a 3-DOF non-redundant PKM that has been patented by Prof. Reymond Clavel and developed at Ecole Polytechnique Federale de Lausanne (EPFL) [21]. Fig. 2
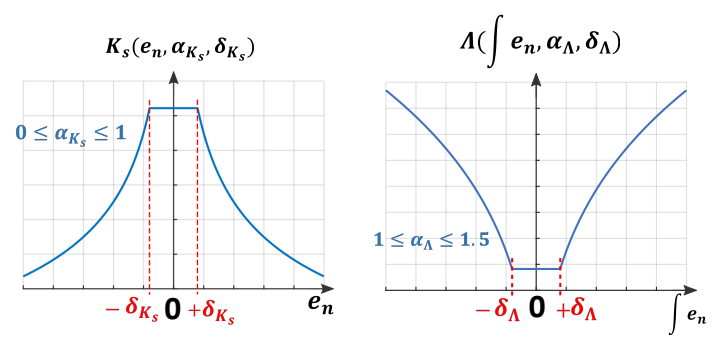

Fig. 1. The evolution of the nonlinear gains with respect to their arguments. 


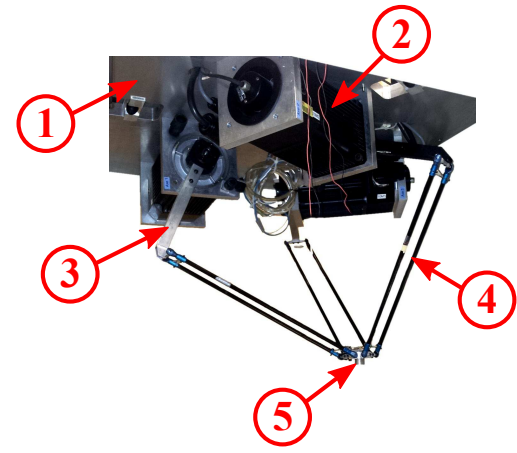

Fig. 2. View of Delta robot including (1): Fixed-base, (2): Actuator, (3): Rear-arm, (4): Forearm, (5): Traveling-plate.

illustrates the a view of the fabricated Delta robot. It consists of a fixed-base linked to a moving platform (travelingplate or end-effector) through three kinematic chains. Each kinematic chain is arranged of an actuator (rotational motor), rear-arm and forearm. The overall assembly allows the enddefector to perform in three translational motions $x, y$ and z. Consider 3-dimensional coordinate vector $X=\left[\begin{array}{lll}x & y & z\end{array}\right]^{T}$ represents the position of the end-effector in the workspace, and the 3-dimensional coordinate vector $q=\left[\begin{array}{lll}q_{1} & q_{2} & q_{3}\end{array}\right]^{T}$ represents the actuated joints configuration.

\section{B. Delta robot dynamics}

The dynamics of Delta PKM are developed in [11] based on the virtual work principal reported with some assumptions for simplification purpose. The traveling-plate is exhibited to two kind of forces: gravitational force and force due to the acceleration. These forces are projected into the joint space to obtain their contribution at the level of motors. From the actuators side, the forces to be considered are the actuators torques, the gravitational forces of the rear-arms and the inertial forces due to the rear-arms rotation.

After applying the virtual work principal and re-arranging the obtained equation, the inverse dynamic equation of Delta robot in joint space is written as follows:

$$
M(q) \ddot{q}+C(q, \dot{q}) \dot{q}+G(q)=\Gamma
$$

where $M(q) \in \mathbb{R}^{3 \times 3}$ is the total mass and inertia matrix of the robot, $C(q, \dot{q}) \in \mathbb{R}^{3 \times 3}$ is the Coriolis and centrifugal forces matrix and $G(q) \in \mathbb{R}^{3}$ is the gravitational forces vector. Note that the dynamics of Delta robot are considered a particular case of the nonlinear system of (1) for $m=3$ and $n=2$.

\section{Control application}

For comparison purpose, both the original rise controller and the proposed time-varying feedback RISE controller are implemented on Delta robot. The tracking error $e_{1}(t)$ and the combined error $e_{2}(t)$ are defined as follows:

$$
e_{1}=q_{d}-q, \quad e_{2}=\dot{e}_{1}+\gamma e_{1}
$$

where $q_{d} \in \mathbb{R}^{3}$ denotes the desired joint position vector, $\gamma \in$ $\mathbb{R}^{3 \times 3}$ is a positive-definite gain matrix added to get more flexibility in tuning process. The standard RISE feedback control law and the proposed one are implemented the same as in sections II and III with $m=3$ and $n=2$.

The tuning process for the proposed controller was done online directly on the real robot following a similar manner for the one proposed in [22] to tune the NPD control gains. The tuning procedure is described as follows: 1) let $\alpha_{K s}=1$, $\alpha_{\Lambda}=1, \lambda=0$ and $\left.\beta=0,2\right)$ increase $\gamma$ and slightly $k_{s}$ starting both from zero until the robot shows good dynamic performance, 3 ) increase the value of $\lambda$ to obtain better tracking performance, then make a trade-off between $\gamma, k_{s}$ and $\lambda, 4)$ find $\left(e_{2}\right)_{\max }$ and $\left(\int e_{2}\right)_{\max }$ values and select their halves as the values of $\delta_{K_{s}}$ and $\delta_{\Lambda}$ respectively, 5) decrease the value of $\alpha_{K s}$ in the interval $[0.5,1]$ and increase the value of $\alpha_{\Lambda}$ in the interval $[1,1.5]$, regulate again the values of $k_{s}$ and $\lambda$ making a compromise among the four values, 6 ) repeat steps (4) and (5) until you obtain the best possible root mean square error, 7) increase $\beta$ unto obtaining better performance index. The appropriate obtained values of control parameters following this procedure are listed in Table I.

One can notice that RISE control law is similar to a 2nd-order SMC where they have used the integral of the signum function to produce continuous signal. Unlike 2ndorder SMC, RISE control is not limited to first-order multiinput systems and can achieve the asymptotic tracking under limited assumptions on the modeling. Through the proper selection of $\beta$ control gain, $\beta \operatorname{sgn}\left(e_{2}\right)$ term can compensate for the unknown and unmeasured nonlinearity [5].

\section{EXPERIMENTAL RESULTS}

The experimental testbed includes the Delta robot shown in Fig. 2 connected to a master computer booting via windows XP equipped with RTX extension. Delta robot is driven by three direct-drive motors that can reach up to 23 $\mathrm{Nm}$ a maximum torque. The control algorithm running at sampling period $1 \mathrm{~ms}$ (frequency $1 \mathrm{KHz}$ ) is developed in $\mathrm{C}++$ language through the Visual Studio software from Microsoft.

To validate the proposed time-varying feedback RISE controller, a comparison study is done with the original RISE controller through experimental scenarios (speed and payload variation) conducted over Delta robot. Root Mean Square Error (RMSE) criteria in Cartesian and joint spaces are used to quantify both controllers as shown below:

$$
\begin{aligned}
R M S E_{x} & =\left(\frac{1}{N} \sum_{i=1}^{N}\left(e_{x}^{2}(i)+e_{y}^{2}(i)+e_{z}^{2}(i)\right)\right)^{1 / 2} \\
R M S E_{J} & =\left(\frac{1}{N} \sum_{i=1}^{N}\left(e_{q_{1}}^{2}(i)+e_{q_{2}}^{2}(i)+e_{q_{3}}^{2}(i)\right)\right)^{1 / 2}
\end{aligned}
$$

TABLE I

CONTROL PARAMETERS

\begin{tabular}{cc|cc|cc}
\hline \multicolumn{2}{c|}{ Standard RISE } & \multicolumn{4}{|c}{ Time-Varying } \\
\hline$\gamma$ & 360 & $\gamma$ & 450 & $\lambda$ & 0.66 \\
$K_{s}$ & 0.35 & $k_{s}$ & 0.35 & $\alpha_{\Lambda}$ & 1.45 \\
$\Lambda$ & 0.66 & $\alpha_{K_{s}}$ & 0.65 & $\delta_{\Lambda}$ & 0.12 \\
$\beta$ & 1.5 & $\delta_{k_{s}}$ & 0.05 & $\beta$ & 2 \\
\hline
\end{tabular}


where $e_{x}, e_{y}, e_{z}$ present the Cartesian position tracking erros along $x, y$, and $z$ axes. $e_{q_{1}}, e_{q_{2}}, e_{q_{3}}$ denote the joints tracking errors. $\mathrm{N}$ is the number of collected samples.

Note that the direct model is used to compute the Cartesian error not for giving the absolute error of the robot, but to indicate the range of errors we can get, since the robot is a translational robot. Knowing that the kinematic calibration may not change that much the range of errors, mainly because the errors between joint and tool space are associated through the Jacobian matrix, the kinematic model is not calibrated. All the offset error effects are cancelled after the derivation. The segment lengths have been measured after manufacturing and they have been adjusted accordingly. The desired trajectory is generated according to pick-and-place motions using semi-ellipses as proposed in [11] which is mainly used for industrial food packaging applications.

\section{A. Scenario 1: nominal case}

In this scenario, the mobile platform of the robot is free of any additional payload and operating at acceleration of 2.5 G. The evolutions of the tracking errors resulting from both controllers in the operational and joint spaces are plotted in Fig. 3 over one cycle of the reference trajectory. It is clear from Fig. 3 that the range of errors resulting from the proposed controller is less than that of the original RISE control. To quantify the improvement of the proposed timevarying feedback RISE controller, the RMSE in Cartesian and joint spaces are calculated using (11) and (12) confirming an enhancement of $18 \%$ in terms of accuracy as shown in Table II. The evolution of the nonlinear feedback gains $\left(K_{s}()+1.\right)$ and $\left(k_{s}+1\right) \Lambda($.$) overall the reference trajectory$ is demonstrated in Fig. 4. The produced adjustments for both gains with passing time give always strictly positive bounded values. The generated control inputs over one cycle of the desired trajectory are always below the actuators limits for both controllers (see Fig. 5).
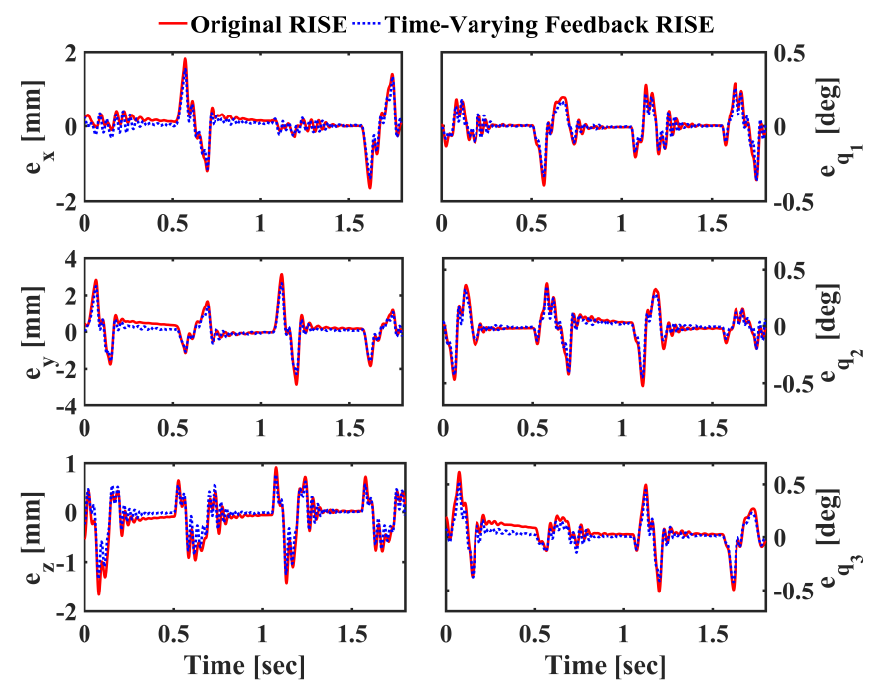

Fig. 3. Scenario 1: Evolution of the Cartesian and joint tracking errors.

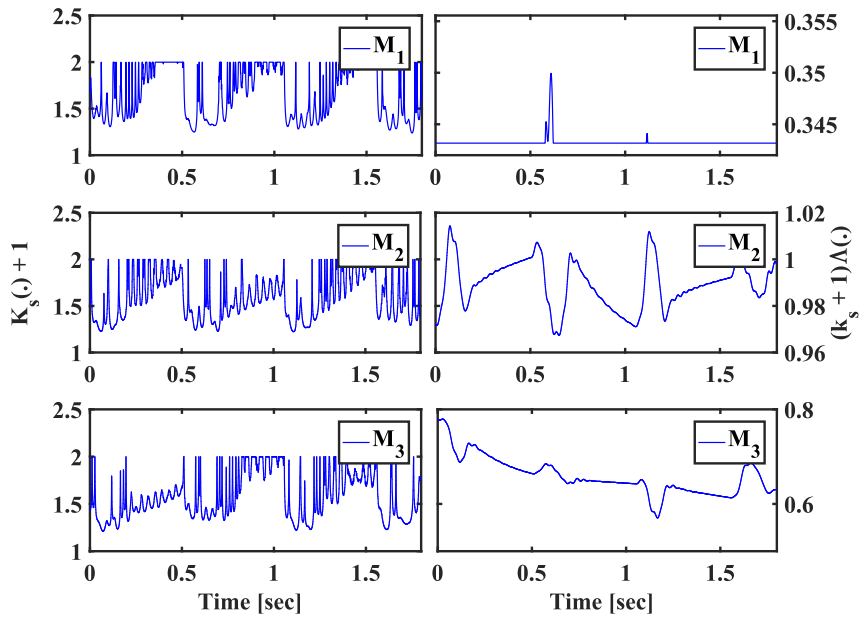

Fig. 4. Scenario 1: Evolution of the nonlinear feedback gains.

B. Scenario 2: Robustness towards speed and payload variation

Delta robot is supposed to operate for pick-and-place applications at high-speed motions. For this, it is important to test the proposed time-varying feedback RISE controller with additional payload and increased acceleration. A mass of $0.225 \mathrm{Kg}$ is attached to the traveling-plate of original mass $0.305 \mathrm{Kg}$ by the means of an electric magnet, and the operating speed is adjusted to an acceleration of $7.5 \mathrm{G}$. From Fig. 6, one can see the relevant improvements obtained by the proposed controller in terms of range of errors in Cartesian and joint spaces. The evaluation criteria for the tracking errors ensure that the proposed time-varying feedback RISE overcomes the original RISE controller with $30.5 \%$ for Cartesian space and $28.3 \%$ for joint space in terms of precision (see Table II). Thanks to the added nonlinearity of time-varying feedback RISE control law, experiments validate its robustness towards different operating conditions as payload and speed variations and more compensation
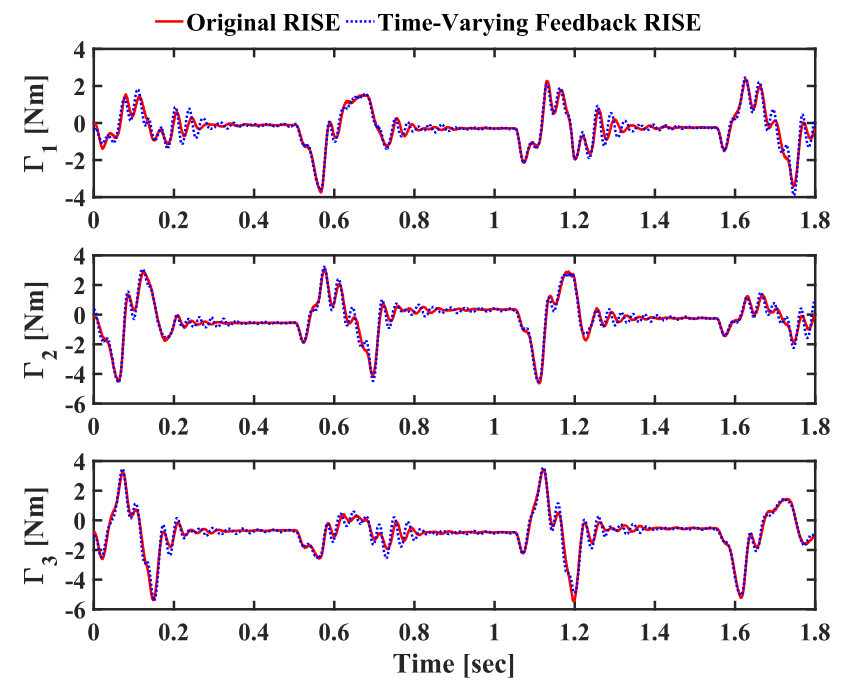

Fig. 5. Scenario 1: Evolution of the control input torques. 
for the abundant nonlinearities in PKMs. The variations of the nonlinear feedback gains along the desired trajectory are represented in Fig. 7. It is obvious that the nonlinear feedback gains remain positive and bounded even with the high changes of the dynamic operating conditions.

Fig. 8 demonstrates the growth of the control input signals overall one cycle of the desired trajectory. It is remarkable from Fig. 8 that the time-varying feedback RISE controller reduces the energy consumption producing less input torques compared to standard RISE controller. To quantify this energy reduction, the following input-torques-based criterion is proposed:

$$
E_{\Gamma}=\sum_{i=1}^{3} \sum_{j=1}^{N}\left|\Gamma_{i}(j)\right|
$$

where $E_{\Gamma}$ is the total positive input torque. In this scenario, the performance in terms of energy consumption is promoted significantly from $1.7692 \times 10^{4} \mathrm{Nm}$ for original RISE to $1.4318 \times 10^{4} \mathrm{Nm}$ for the new time-varying feedback RISE with a reduction of $19.1 \%$. Note that the control signals for both controllers evolve within the safe range of the actuators capabilities.

\section{Performance index versus operating acceleration}

In this section, the operating acceleration is increased gradually starting from $2.5 \mathrm{G}$ reaching up $10 \mathrm{G}$. Both controllers have been treated with the same manner in two cases: with and without additional payload $(0.225 \mathrm{Kg})$. Fig. 9(a) and Fig. 9(b) are two bar graphs showing the variation of $R M S E_{x}$ in $(\mathrm{mm})$ with respect to the operating acceleration in $(\mathrm{G})$ in case of no added payload and payload of $0.225 \mathrm{Kg}$ respectively. The quantified improvement of the new timevarying feedback RISE controller at each acceleration is written at the top of the corresponding column. It can be clear that the performance of time-varying feedback RISE is better than that of standard RISE in all cases. However, the gathered improvements of the proposed controller are much
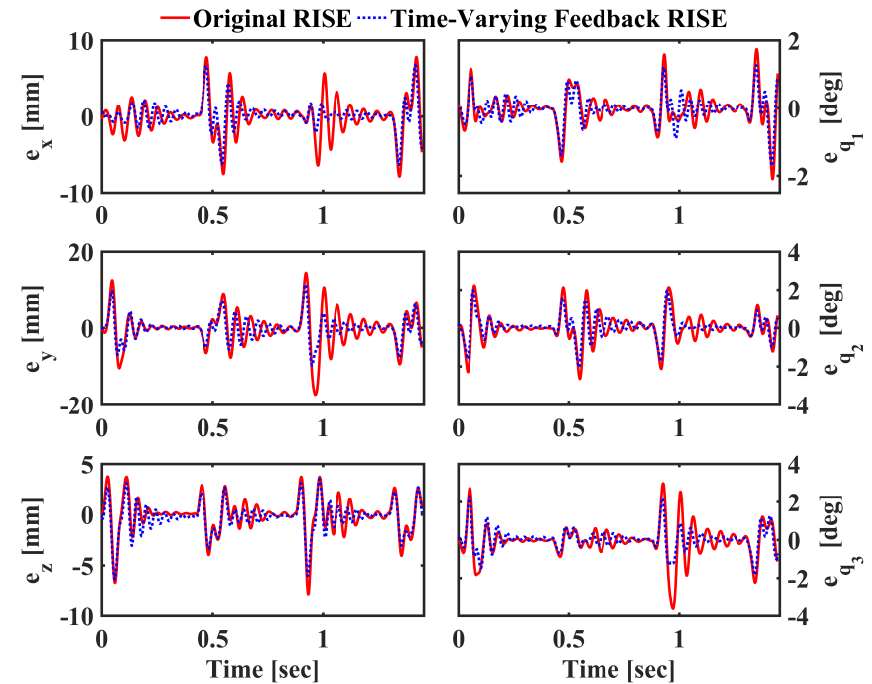

Fig. 6. Scenario 2: Evolution of the Cartesian and joint tracking errors.
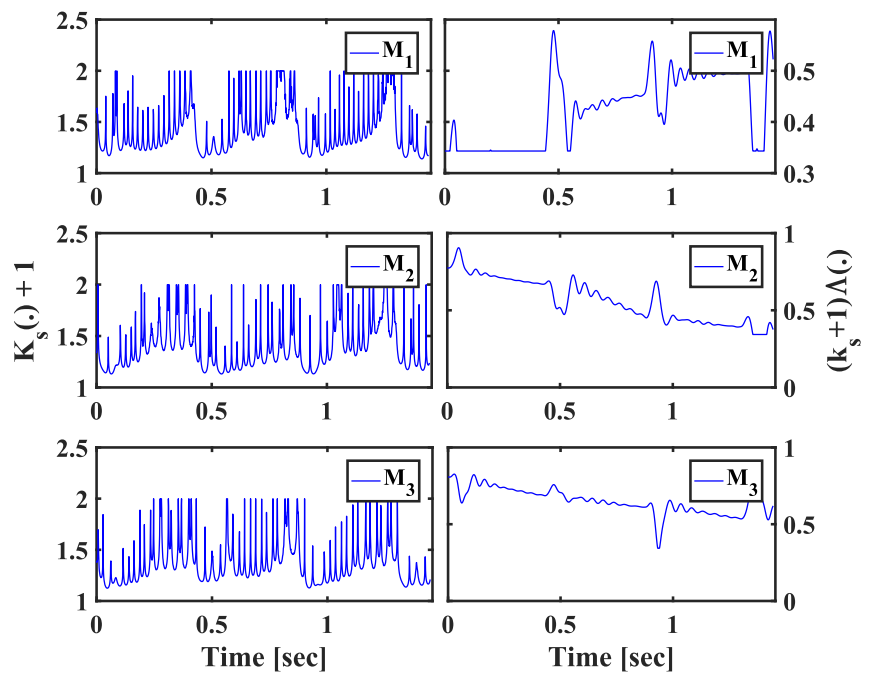

Fig. 7. Scenario 2: Evolution of the nonlinear feedback gains.

better in the case of added payload than that of no payload. It is verified that the proposed nonlinear control law based on time-varying feedback gains is much appropriate for nature of PKMs especially when operating at high dynamics such as payload and acceleration. It is noticeable that at acceleration of $10 \mathrm{G}$ in the case of added payload, the generated joint errors override 10 degrees, the specified safety margins for the robot to turn off, with RISE controller. While timevarying feedback RISE controller produces acceptable errors always within the defined safety margins.

\section{CONClusions AND Future Work}

In this work, the standard RISE control strategy has been extended to a new time-varying feedback RISE control law. This approach takes the advantage of the nonlinear feedback gains which are adjusted online with respect to the system states, and inserts them instead of the static feedback gains of standard RISE controller. The validation
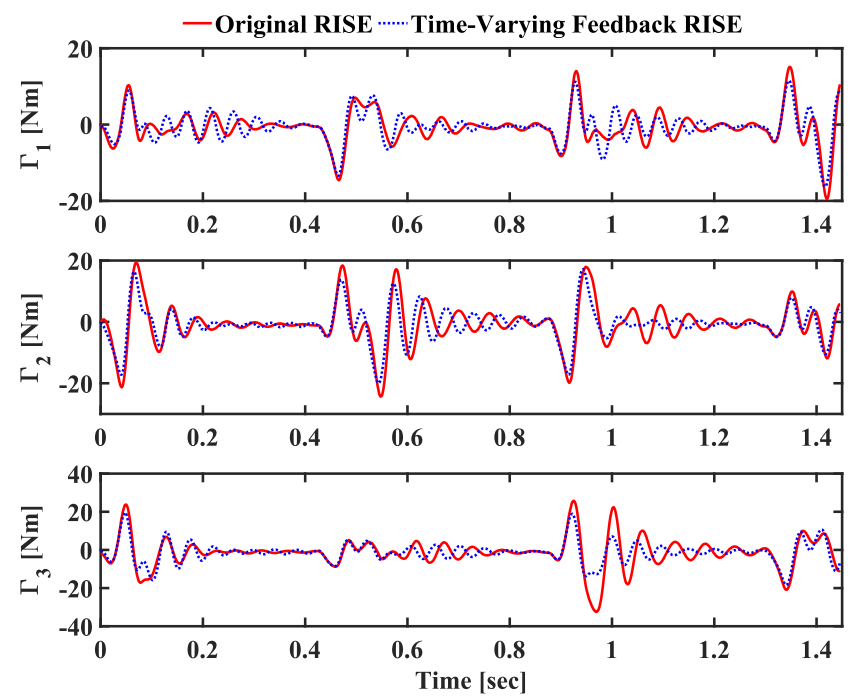

Fig. 8. Scenario 2: Evolution of the control input torques. 
TABLE II

CONTROL PERFORMANCE EVALUATION

\begin{tabular}{lccc}
\hline Scenario & Control & $R M S E_{x}[\mathbf{m m}]$ & $R M S E_{J}[\mathbf{d e g}]$ \\
\hline \multirow{3}{*}{ Scenario 1 } & RiSE & 0.993 & 0.2341 \\
& Improve-Varying Feedback RISE & 0.8129 & 0.192 \\
& RISE & $\mathbf{1 8 . 1} \%$ & $\mathbf{1 8} \%$ \\
Scenario 2 & Time-Varying Feedback RISE & 5.3985 & 1.2577 \\
& Improvements & $\mathbf{3 0 . 5} \%$ & 0.9012 \\
& & $\mathbf{2 8 . 3} \%$ \\
\hline
\end{tabular}

of the proposed control law was performed through real-time experiments on a non-redundant 3-DOF parallel robot called Delta. Experimental results approved that the performance of the proposed control solution overcomes the standard RISE in terms of precision, high-speed motions and robustness towards payload variation. Furthermore, It requires less control efforts when operating at high dynamic conditions regarding payload and acceleration. As a future work, this paper can be extended with the stability analysis of the proposed controller. Furthermore, incorporating the dynamics of the parallel robot within an adaptive model-based closedloop controller can enhance the general performance of the proposed control approach.

\section{REFERENCES}

[1] J.-J. E. Slotine and W. Li, Applied Nonlinear Control. Englewood Cliffs, New Jersey: Prentice Hall, 1991.

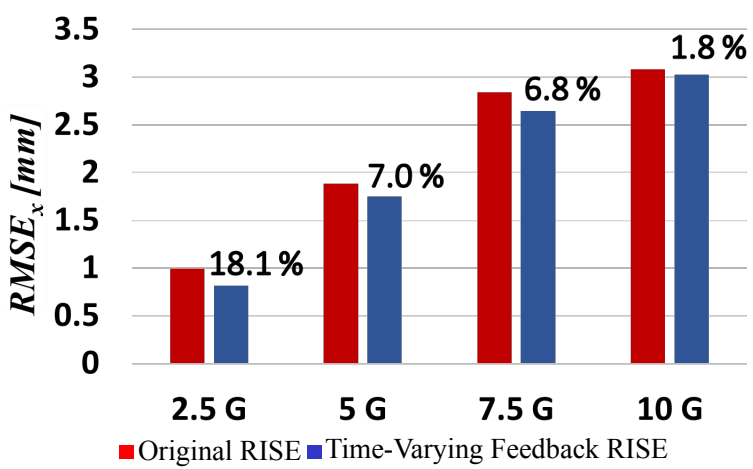

(a) No payload

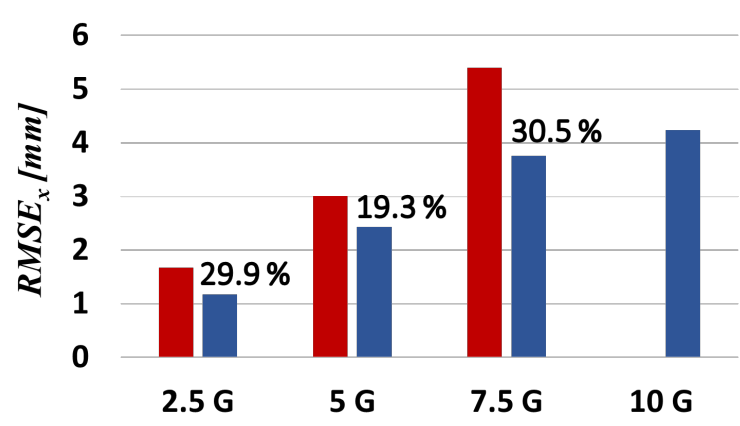

- Original RISE — Time-Varying Feedback RISE

(b) Payload of $0.225 \mathrm{Kg}$

Fig. 9. Clustered column chart of $R M S E_{x}$ versus acceleration
[2] H. Jingqing, "A new type of controller: Nlpid," Control and Decision, vol. 9, no. 6, pp. 401-407, 1994.

[3] H. Jianbo and S. Manhong, "Robust gain-scheduling control for a class of uncertain nonlinear systems with exogenous signals," in Proc. IEEE 6th World Congress on Intelligent Control and Automation, Dalian, China, June 21-23, 2006, pp. 2234-2238.

[4] L. Ling and D. Hao, "A new hyperchaotic system control via adaptive feedback design with an uncertain parameter," in Proc. IEEE 32nd Chinese Control Conference, Xi' an, China, July 26-28, 2013, pp. 840843.

[5] B. Xian, M. de Queiroz, and D. Dawson, "A continuous control mechanism for uncertain nonlinear systems," in Optimal Control, Stabilization and Nonsmooth Analysis. Berlin, Heidelberg: Springer, 2004, vol. 301, pp. 251-264.

[6] N. Fischer, D. Hughes, P. Walters, E. Schwartz, and W. Dixon, "Nonlinear rise-based control of an autonomous underwater vehicle," IEEE Transactions on Robotics, vol. 30, no. 4, pp. 845-852, August 2014.

[7] N. Fischer, Z. Kan, R. Kamalapurkar, and W. Dixon, "Saturated rise feedback control for a class of second-order nonlinear systems," IEEE Transactions on Automatic Control, vol. 59, no. 4, pp. 1094-1099, April 2014.

[8] N. Kamaldin, S. Chen, C. Kong, C. Teo, and K. Tan, "Adaptive parameter and gain rise control of a flexure-based dual-drive ' $h$ ' gantry," in Proc. IEEE International Conference on Advanced Intelligent Mechatronics (AIM), Alberta, Canada, 12 July-15 July, 2016 pp. 1240-1245.

[9] J. Yao, W. Deng, and Z. Jiao, "Rise-based adaptive control of hydraulic systems with asymptotic tracking," IEEE Transactions on Automation Science and Engineering, vol. 14, no. 3, pp. 1524-1531, July 2017.

[10] M. Bennehar, A. Chemori, and F. Pierrot, "A novel rise-based adaptive feedforward controller for redundantly actuated parallel manipulators," in Proc. IEEE International Conference on Intelligent Robots and Systems (IROS), Chicago, IL, USA, Sept.14-18, 2014, pp. 2389-2394.

[11] M. Bennehar, A. Chemori, M. Bouri, L. Jenni, and F. Pierrot, "A new rise-based adaptive control of pkms: Design, stability analysis and experiments," International Journal of Control, vol. 91, no. 3, pp 593-607, 2018.

[12] G. Chaudhary and J. Ohri, "3-dof parallel manipulator control using pid controller," in Proc. IEEE 1st International Conference on Power Electronics, Intelligent Control and Energy Systems, Delhi, India, July4-6, 2016, pp. 1-6.

[13] Y. Su, B. Duan, and C. Zheng, "Nonlinear pid control of a six-dof parallel manipulator," IEE Proceedings-Control Theory and Applications, vol. 151, no. 1, pp. 95-102, January 2004.

[14] A. Codourey, "Dynamic modeling of parallel robots for computedtorque control implementation," The International Journal of Robotics Research, vol. 17, no. 12, pp. 1325-1336, December 1998.

[15] M. Rachedi, B. Hemici, and M. Bouri, "Design of an ho controller for the delta robot: experimental results," Advanced Robotics, vol. 29, no. 18, pp. 1165-1181, 2015.

[16] H. Saied, A. Chemori, M. E. Rafei, C. Francis, and F. Pierrot, "Actuator and friction dynamics formulation in control of pkms: From design to real-time experiments," in Proc. IEEE International Conference on Intelligent Robots and Systems (IROS), Madrid, Spain, 01 Oct-5 Oct, 2018, pp. 5634-5639.

[17] G. S. Natal, A. Chemori, and F. Pierrot, "Dual-space control of extremely fast parallel manipulators: Payload changes and the $100 \mathrm{~g}$ experiment," IEEE Transactions on Control Systems Technology, vol. 23 , pp. 1520-1535, July 2015.

[18] M. Bennehar, G. El-Ghazaly, A. Chemori, and F. Pierrot, "A novel adaptive terminal sliding mode control for parallel manipulators: Design and real-time experiments," in Proc. IEEE International Conference on Robotics and Automation (ICRA), Singapore, Singapore, June29 May-3 June, 2017, pp. 6086-6092.

[19] G. S. Natal, A. Chemori, and F. Pierrot, "Nonlinear control of parallel manipulators for very high accelerations without velocity measurement: Stability analysis and experiments on par2 parallel manipulator,' Robotica, vol. 34, pp. 43-70, Jan 2016.

[20] M. Bennehar, A. Chemori, and F. Pierrot, "A new revised desired compensation adaptive control for enhanced tracking: application to ra-pkms," Advanced Robotics, vol. 30, no. 17-18, pp. 1199-1214, June 2016.

[21] R. Clavel, "Device for the movement and positioning of an element in space," Switzerland Patent 4976582, dec 11, 1990.

[22] W. Shang, S. Cong, Z. Li, and S. Jiang, "Augmented nonlinear pd controller for a redundantly actuated parallel manipulator," Advanced Robotics, vol. 23, no. 12-13, pp. 1725-1742, 2009. 